\title{
Salt Modulates Oligomerization Properties of hRad51 and hRad52 Proteins
}

\author{
Kamakshi Balakrishnan ${ }^{\dagger}$ Neeraja M. Krishnan ${ }^{\dagger}$ and Basuthkar J. Rao* \\ Department of Biological Sciences, Tata Institute of Fundamental Research, Homi Bhabha Road, Colaba, Mumbai-400 \\ 005, India
}

\begin{abstract}
Human Rad52 (hRad52) and Rad51 (hRad51) proteins are important components of homologous recombination machinery involved in DNA double strand break repair. hRad52 subunits oligomerize to form rings, which are further believed to stack one over another giving rise to higher order structures. Such structures bind the ends of duplex DNA to bring about DNA end joining. hRad51 exists in the native state as oligomeric rings and monomerizes to interact with the DNA. In our current study, we report disruption and solubilization of hRad52 aggregates and higher order aggregation of hRad51 molecules at high salt $(\mathrm{KCl})$ concentration. Computational analysis of the crystal structure available for $\mathrm{N}$-terminal 212 amino acids of hRad52 protein reveal a dense unique distribution of salt bridges, not only between adjacent but also between penultimate subunit neighbors which perhaps contribute to stabilization of hRad52 oligomeric rings. Our results suggest that disruption of inter-subunit salt bridges and thereby perturbation of interaction between individual monomers as the underlying mechanism for salt mediated monomerization of hRad52 protein. The crystal structure of Rad51 on the other hand lacks such dense salt-bridge connectivity suggesting that salt-mediated monomerization is a feature of proteins with dense salt-bridge networks. Salt brings together the hydrophobic surface residues of hRad51 in a process termed as "salting out" resulting in aggregation of hRad51 molecules. Given the functional relevance of oligomeric hRad52 and monomeric hRad51 in homologous recombination mediated repair, our findings imply that salt regulates the oligomerization status of these repair proteins, and thereby, their functions respectively.
\end{abstract}

Keywords: hRad52, hRad51, salt bridge, monomerization, aggregation, oligomers, $\mathrm{KCl}$.

\section{INTRODUCTION}

Human Rad52 (hRad52) and Rad51 (hRad51) are core components of the homologous recombination machinery that repairs DNA double-strand breaks inside mammalian cells. hRad52 is a key recombination mediator protein that plays a significant role in the initial steps of DNA double strand break repair via specific interactions with proteins of homologous recombination machinery, particularly with Rad51 [1-7] and Replication protein A [8, 9]. Genetic studies point towards roles of Rad52 in Rad51recombinase dependent [3] and -independent recombination pathways $[10,11]$. Rad52 also catalyzes homologous pairing between DNA sequences independent of $\operatorname{Rad} 51$ as observed by ssDNA annealing [12-14] and DNA strand exchange [15, 16] assays.

Electron microscopy (EM), dynamic light scattering (DLS) and gel filtration studies reveal that hRad52 protein exhibits two modes of self association namely, formation of ring shaped oligomers and formation of higher order complexes of these oligomeric rings [17-21]. Such higher order aggregates have been shown to promote hRad52 binding to DNA ends $[18,19]$ and are suggested to be relevant in vivo, where Rad52 can simultaneously interact with more than one DNA molecule [20]. It has been observed in Saccharomyces cerevisiae that the naturally

*Address correspondence to this author at the Department of Biological sciences, Tata Institute of Fundamental Research, Homi Bhabha Road, Colaba, Mumbai-400 005, India; Tel: 091-22-22782606; Fax: 091-2222804610; E-mail: bjrao@ tifr.res.in;

URL: http: //www.tifr.res.in/ dbs/faculty/bjr/

†Equal Contribution weak NLS signal in monomeric form of $\operatorname{Rad52}$ gets amplified in the oligomerized $\operatorname{Rad52}$ form, due to the additive effect of NLS from monomeric subunits, thereby driving efficient nuclear localization of $\operatorname{Rad} 52$ to bring about repair [22]. The full length $h$ Rad52 protein (1-418 residues) that catalyzes homologous pairing [21] predominantly forms heptameric rings [24], whereas the N-terminal (1-192 residues) and C-terminal (218-418 residues) domains associate with formation of ring shaped oligomers and higher order self-association events such as stacking of oligomeric rings, respectively [17]. Moreover, the $\mathrm{N}$-terminal fragment is identified as the catalytic domain for homologous pairing [24] and forms filamentous complexes containing either stacked rings or rings arranged in a side-by-side manner, in the presence of ssDNA [21]. The crystal structure of the ring formed by this $\mathrm{N}$ - terminal domain fragment (1-212) verifies predictions from DLS and sedimentation equilibrium studies of an undecameric (eleven subunit) organization [24].

hRad51, the other core component of recombination machinery is the central recombinase protein that catalyzes defining mechanistic steps such as homologous pairing and strand exchange in recombination [25]. hRad51 has structural and functional homology to classical recombinase, E.coli RecA [26, 27] and is very well conserved among eukaryotes [28, 29] as well as in archaea [30, 31]. hRad51 shares $46 \%$ overall sequence identity with archaeal Rad51[31]. hRad51 binds ssDNA as well as dsDNA efficiently and exhibits DNA dependent ATPase activity[3234]. Crystal structure [31, 35], EM [36] and biochemical studies reveal that Rad51 and related recombinases exist in (i) ring forms in absence of DNA [34, 37-40] (ii) and helical filamentous forms in presence of DNA [40-43]. The former 
ring-like recombinase structures do not appear to be the form of the protein that is active during the strand exchange phase of homologous recombination [31, 37, 44, 45]. It is further conceived that oligomeric Rad51 rings are broken down into monomers in presence of DNA. These monomers are then suggested to polymerize on DNA substrate to result in an active helical nucleo-protein filament [43, 45-49]. The resultant biologically active Rad51-DNA filament catalyzes pairing and strand exchange between homologous DNA molecules, an essential step in homologous recombination [42-45]. Full-length RAD51 homologs have proven challenging to crystallize, likely due to flexiblity in their two-domain structures and a tendency to form polymers [31]. Crystal structures are however, available for human Rad51ATPase domain [50] and also for ADP bound [51] non-hydrolyzable ATP analog (AMP-PNP, ATP $\gamma \mathrm{S}$ )/ Potassium bound forms of archaeal Rad A, Methanococcus voltae (Mv RadA) [52, 53]. X-ray crystal structure available for polymeric full-length Rad51 homolog from the thermophilic archaeaon Pyrococcus furiosus (Pf Rad51) has revealed how Rad51 proteins assemble into inactive heptameric rings and active DNA-bound filaments matching three-dimensional electron microscopy reconstructions [31]. The study shows further structural details including a polymerization motif (PM) involving an interdomain linker key for quaternary assembly [31].

Rad51 genetically and physically interacts with Rad52. Rad52 functions in synergy with Rad51and stimulates Rad51 mediated functions in homologous recombination such as generation of Rad51-DNA nucleoprotein filament, homologous pairing and strand exchange between homologous DNA molecules $[1,3,54,55]$. Both proteins co-localize in distinct repair proficient nuclear foci in response to DNA damage [56]. Rad52 is required for such foci formation during meiosis [57]. In light of such demonstrated functions of Rad52 in a variety of Rad51 activities, we have studied the effects of salt on aggregation status of hRad52 and hRad51 proteins. In this correspondence, we report that salt $(\mathrm{KCl})$ at high concentration mediates disruption and monomerization of hRad52 aggregates. On the other hand addition of salt in case of hRad51 exerts an opposing effect, namely aggregation of hRad51 molecules. Computational analysis of crystal structure data available for $\mathrm{N}$-terminal hRad52 (1-212) (PDB ID: 1KN0) [24] and full length archaeal polymeric Rad51 (PDB ID: 1PZN) [31] further revealed presence of a dense unique distribution of salt bridges between adjacent and penultimate subunit neighbors in hRad52 undecamers and a lack of the same (such dense salt bridge connectivity between Rad51 subunits) in case of $P f$ Rad51. Our findings imply disruption of inter-subunit salt bridges as the underlying mechanism for salt mediated disruption of hRad52 aggregates. On the contrary, in case of hRad51, salt brings about aggregation of hRad51 due to hydrophobic collapse where the hydrophobic residues at the interacting surfaces of monomers come together to result in larger structures.

\section{MATERIALS AND METHODOLOGY}

\section{Materials}

The hRad52 over expressing clone was kindly provided by Dr. Steve West (Cancer Research, UK) and the protein was purified as described [58]. The hRad51 over expressing clone was kindly provided by Prof. Hitoshi Kurumizaka and the protein was purified as described [59]. $\mathrm{KCl}$ was purchased from SISCO Laboratories, INDIA.

\section{Centrifugation Assay}

hRad52 protein $(2 \mu \mathrm{M})$ or hRad51 $(2 \mu \mathrm{M})$ was incubated in binding buffer $\mathrm{R}$ [30 mM Tris- $\mathrm{HCl}$ (pH 8.0), 1mM DTT, $1 \mathrm{mM} \mathrm{MgCl}_{2}$ ] with or without $\mathrm{KCl}$ at $37^{\circ} \mathrm{C}$ for 30 minutes. Samples were centrifuged with eppendorf centrifuge at 16, $100 \mathrm{~g}$ for 10 minutes at room temperature. The supernatant and pellet fractions were separated and heated in Laemmli buffer at $90^{\circ} \mathrm{C}$ for 10 minutes and analyzed by SDS PAGE (12\% acrylamide) followed by Coomassie staining. For native gel analysis, supernatant and pellet fractions were run on a $6 \%$ polyacrylamide gel in TBE (Tris Borate EDTA) buffer at 120 volts for $6 \mathrm{hrs}$ at $\sim 23^{\circ} \mathrm{C}$ and visualized by silver staining.

\section{Computational Analysis}

We used WHAT IF tool (http: //swift.cmbi.ru.nl/servers/ $\mathrm{html} /$ index.html) to compute salt bridges between individual subunits of hRad52 and hRad51 oligomers. Crystal structure of hRad52 (PDB ID: 1KN0) comprising 1-212 amino acid residues from the $\mathrm{N}$-terminal fragment and full length archaeal Rad51 (PDB ID: 1PZN), was provided as respective inputs.

\section{RESULTS}

\section{Salt at High Concentration Monomerizes hRad52 Aggregates and Aggregates hRad51 Molecules}

hRad52 protein was centrifuged in absence or presence of varying concentrations of $\mathrm{KCl}$ and resultant supernatant and pellet fractions were subjected to native PAGE (Fig. 1 (i)) or SDS PAGE (Fig. 1 (ii)). hRad52 in its native state is a highly aggregated protein and is known to exist as a distribution composed predominantly of oligomers and to a minor extent, also monomers [59]. Accordingly, we recover hRad52 protein upon centrifugation, entirely in pellet fraction as a distribution of predominantly higher order aggregates that did not enter gel (data not shown) and fewer monomers that enter the gel in native conditions (lane p1, Fig. 1 (i)). Since the aggregated higher oligomeric forms are retarded in the well, there is a high possibility that they can get dispersed over time under native gel electrophoresis conditions. This possibility renders these well-retarded forms unreliable for comparisons across lane conditions. We, therefore, focus only on those reliable and comparable fractions that enter the gel and form discrete bands. We find that addition of salt $(\mathrm{KCl})$ at concentrations $>25 \mathrm{mM}$ results in an increase in the proportion of hRad52 monomers recovered in supernatant fractions (lanes s2-s6, (Fig. 1) (i)). We confirm this by using commercially available low molecular weight marker (last lane, (Fig. 1) (i)) that the resultant protein species are indeed monomers as the position of protein band on gel corresponds to $\sim 55 \mathrm{KDa}$ which is the expected molecular weight of hRad52 monomer. In addition, SDS PAGE analysis also show an increase in recovery of hRad52 protein levels in supernatant fractions (lanes s2-s6, 


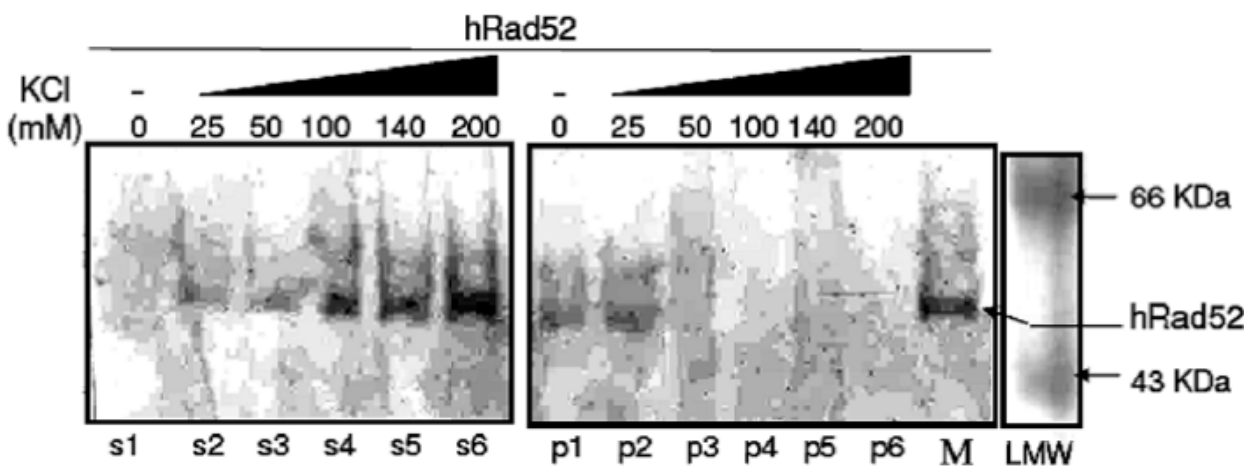

(i)

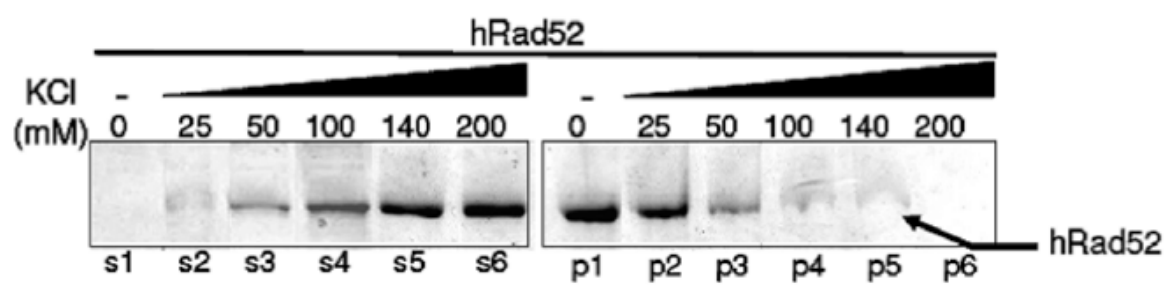

(ii)

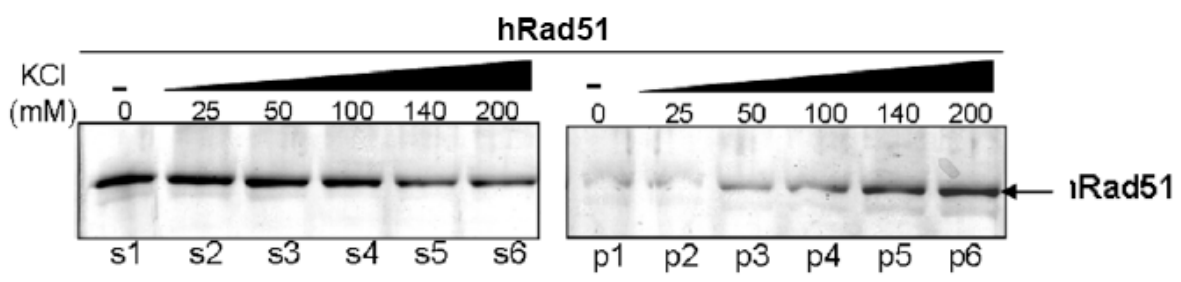

(iii)

Fig. (1). Salt mediated monomerization of hRad52 aggregates

$\mathrm{hRad} 52$ protein $(2 \mu \mathrm{M})$ was incubated in presence of increasing concentrations of $\mathrm{KCl}$ in binding buffer $\mathrm{R}$ at $37^{\circ} \mathrm{C}$ for 12 minutes, followed by centrifugation assay (see Methods) and analyses of proteins by Native PAGE (i) and SDS-PAGE (ii). Lanes s1-s6 and p1-p6 in (i) and (ii) represent supernatant and pellet fractions for increasing concentrations of salt respectively. hRad51 protein $(2 \mu \mathrm{M})$ following salt titration was subjected to centrifugation assay and SDS-PAGE analysis (iii) as explained above. Lane M of Fig. (1(i)) corresponds to marker (pure hRad52 protein) that is not subjected to centrifugation. Last lane of Fig. (1(i)) corresponds to low molecular weight marker (LMW).

Fig. 1 (ii)) and concomitant depletion of the same in pellet fractions following addition of $\mathrm{KCl}$ in the concentration range of $25 \mathrm{mM}-200 \mathrm{mM}$ (lanes p2-p6, Fig. 1 (ii)). At about $200 \mathrm{mM} \mathrm{KCl}$, the protein is completely recovered in supernatant fraction (lane s6, (Fig. 1) (i) and (ii)) with no protein in pellet fraction (lane p6, Fig. 1 (i) and (ii)). The common salt regimes (25 mM-200 mM) in which we observe solubilization of hRad52 using SDS as well as native PAGE analyses depict that salt $(\mathrm{KCl})$ in this concentration range induces monomerization of hRad52 aggregates. hRad51 on the other hand showed exactly the opposite trend upon addition of salt. Unlike hRad52, hRad51 protein is not highly aggregated and it is soluble in its native state and therefore recovered largely in supernatant fraction following centrifugation (lane s1, Fig. 1 (iii)). However we find that following addition of salt in the concentration range (50- 200) $\mathrm{mM}$, hRad51 starts appearing in pellet fractions (lanes p3-p6, Fig. 1(iii)) following centrifugation as analyzed on an SDS-PAGE gel. We did not perform native gel analysis for hRad51 as we are monitoring salt induced aggregation and not monomerization. Further recovery of hRad51 in pellet fractions following centrifugation itself is suggestive of aggregation. Hence we carried out only SDSPAGE analysis of supernatant and pellet fractions of hRad51 following addition of salt.

Crystallized hRad52 (1-212) Exhibits a Unique Dense Distribution of Salt Bridges between Individual Subunits while Crystallized PfRad51 Lacks such Salt Bridge Connectivity

We employed the crystal structure data available for the homologous pairing domain of hRad52 undecamer (PDB ID: $1 \mathrm{KN} 0$ ), comprising of $\mathrm{N}$-terminal 212 amino acids to compute salt bridges between individual subunits. An inter- 

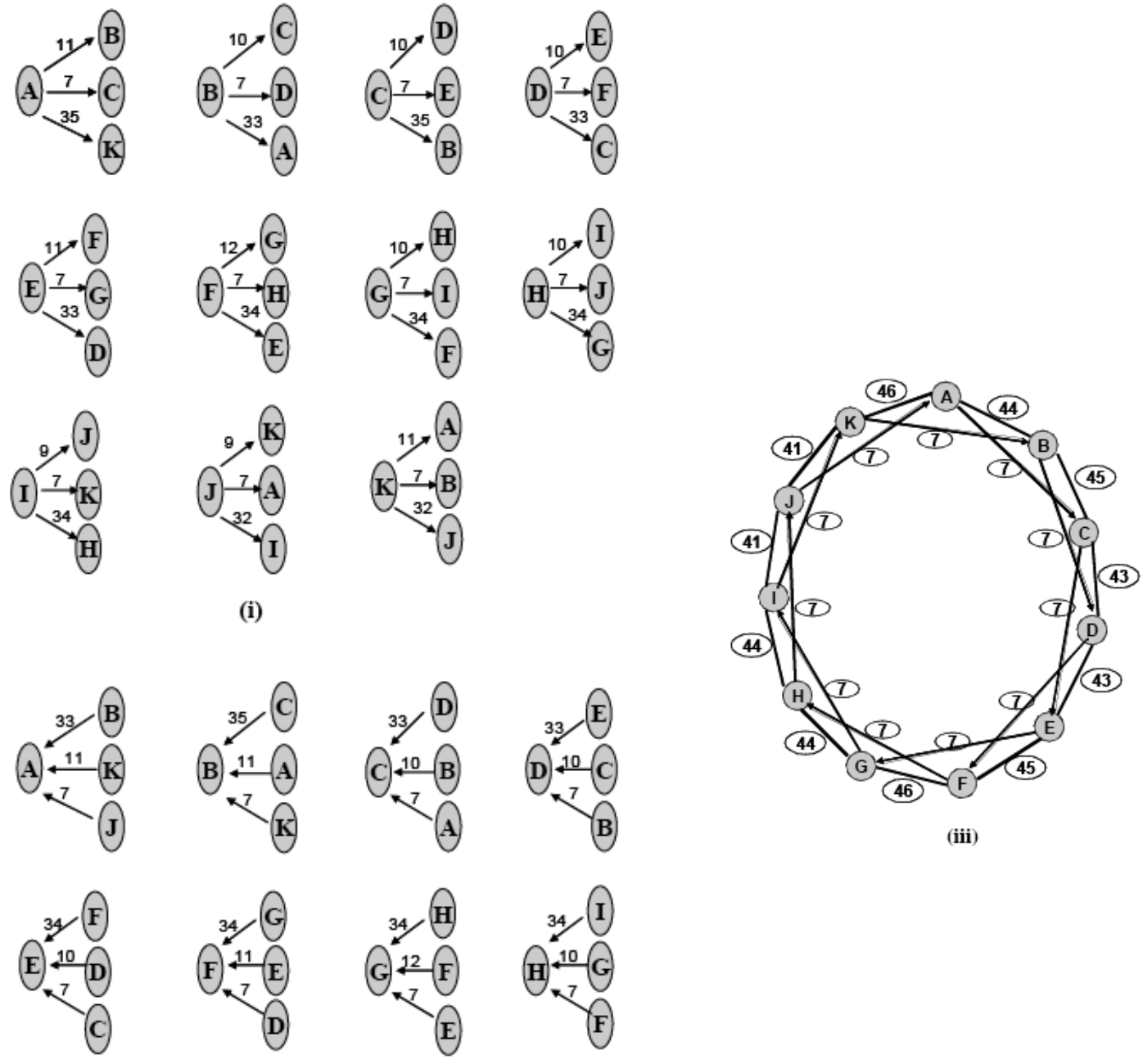

(iii)
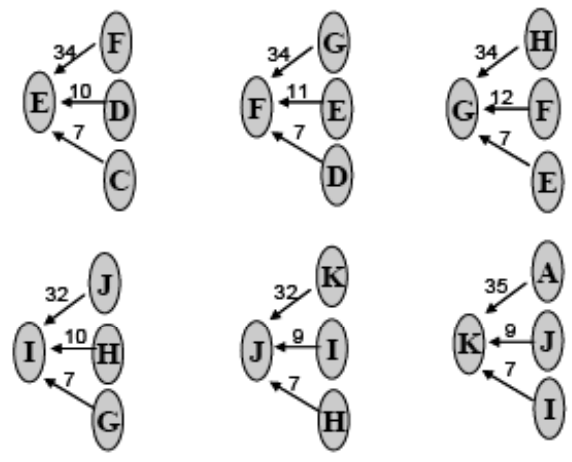

(ii)

Fig. (2). Salt bridge connectivity between hRad52 subunits

The eleven sequential subunits of hRad52 undecamer are annotated as A-K. The inter-subunit salt bridges computed using WHAT IF tool (http: //swift.cmbi.ru.nl/servers/html/index.html) are represented individually as (i) donor and (ii) acceptor maps, and as (iii) the total connectivity map. The numbers above each connection indicate the number of salt bridges. An arrow, wherever represented, indicates the direction of salt bridge, from donor to acceptor subunit. A solid line, wherever represented, indicates the sum of salt-bridges between the donor and acceptor subunits, in both directions.

atomic distance of $<=7 \mathrm{~A}^{\circ}$ between charged residues (donor and acceptor) determines presence of salt-bridges. A donor subunit establishes contacts via Glutamate/Aspartate residues with Histidine/Lysine/Arginine residues of the acceptor subunit. The PDB data was fed to the WHAT-IF tool (see Methods) to compute all potential salt bridges between individual monomers in hRad52 (1-212) oligomer. If eleven sequential monomers in hRad52 undecamer are annotated as A-K (Fig. 2), the donor-acceptor salt bridge pattern can be described as follows: Subunit A (n) donates electrons to its succeeding $B(n+1)$, preceding $K(n-1)$ adjacent subunits, and subunit next to adjacent succeeding subunit $C(n+2)$. This pattern repeats with all subunits (Fig. 2 (i)). Conversely, each subunit (n) is accepting electrons not only from its immediate adjacent neighbors $(n+1$ and $n-1)$ but also from (n-2) subunit (Fig. 2 (ii)). The over all intersubunit connectivity can be described using the total number of electrostatic interactions (electron donation + electron acceptance) as depicted in (Fig. 2 (iii)). Similar computations were performed for $P f \operatorname{Rad} 51$ oligomer by feeding its PDB data into WHAT-IF tool. The seven sequential subunits of Rad51 heptamer are annotated as A-G. Pf Rad51 heptamer exhibits the following salt-bridge pattern. Here each subunit (n) donates as well as receives electrons from its succeeding $(n+1)$ and preceding subunit (n-1) neighbors. The intersubunit salt bridges computed using WHAT IF tool are 


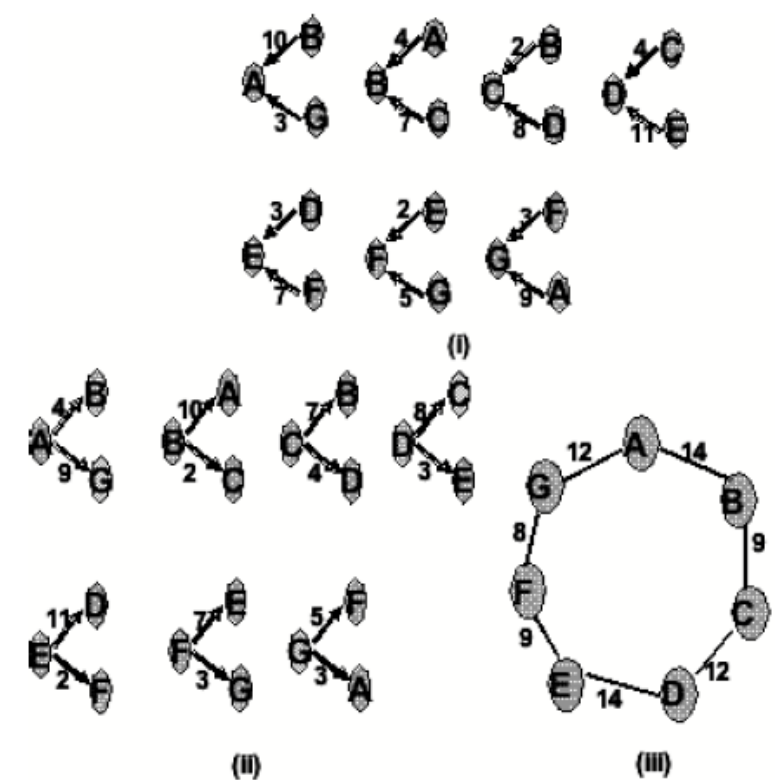

Fig. (3). Salt bridge connectivity between Rad51 subunits

The seven sequential subunits of Pf Rad51 heptamer are annotated as A-G. The inter-subunit salt bridges computed using WHAT IF tool (http: //swift.cmbi.ru.nl/servers/html/index.html) are represented individually as (i) donor and (ii) acceptor maps, and as (iii) the total connectivity map. The numbers above each connection indicate the number of salt bridges. An arrow, wherever represented, indicates the direction of salt bridge, from donor to acceptor subunit. A solid line, wherever represented, indicates the sum of salt-bridges between the donor and acceptor subunits, in both directions.

represented individually as (Fig. 3(i)) donor and (Fig. 3 (ii)) acceptor maps, and as (Fig. 3(iii)) the total connectivity map.

\section{Effect of Salt on Oligomerization Status of hRad52 and hRad51 Proteins}

We propose that addition of $\mathrm{KCl}$ probably disrupts intersubunit electrostatic interactions in hRad52 oligomer (Fig. 4(ii)) and thereby results in generation of hRad52 monomers (Fig. 4(iii)). Conversely in case of hRad51, addition of salt induces aggregation of hRad51 molecules by a phenomenon commonly termed as "salting-out". Here inclusion of salt at high concentration brings about hydrophobic collapse where the hydrophobic residues at the interacting surfaces of monomers come together to result in larger structures.

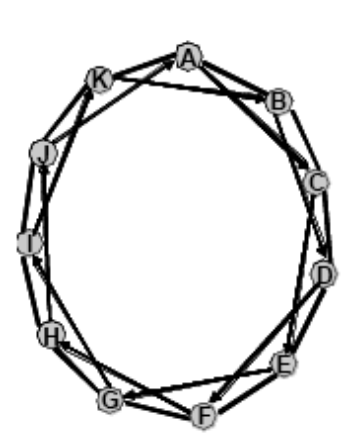

(i)
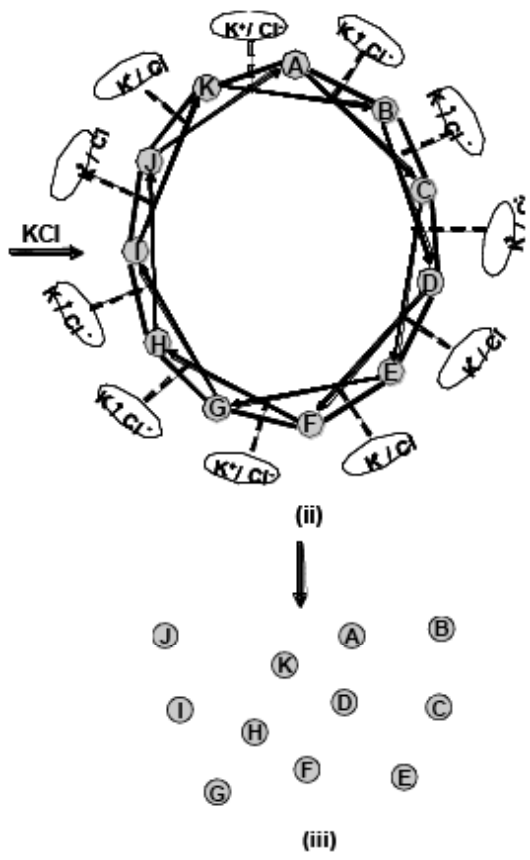

Fig. (4). Schematic representing salt (KCl) mediated monomerization of hRad52 oligomer $\mathrm{KCl}$ disrupts inter-subunit salt bridges (ii) in hRad52 oligomer (i) and generates monomers (iii). 


\section{DISCUSSION}

In our current study we find that salt $(\mathrm{KCl})$ at high concentration $(25 \mathrm{mM}-200 \mathrm{mM})$ results in an increase in the proportion of hRad52 monomers (Fig. 1(ii)) and thereby solubilizes hRad52 protein resulting in their recovery in supernatant fractions following centrifugation (Fig. 1(i) and (ii)). Similar effects have been observed by our group [45] with $\sim$ ten fold lower concentration of $\mathrm{MgCl}_{2}$. The different concentration regimes of $\mathrm{MgCl}_{2}$ and $\mathrm{KCl}$, at which we observe monomerization of hRad52 could be justified by balancing effects of ionic radii and charge of $\mathrm{Mg}^{2+}$ and $\mathrm{K}^{+}$ cations, respectively.

\section{Salt Disrupts Inter-Subunit Salt Bridges in hRad52 Oligomers}

Salt bridges have long been associated with strengthening inter-subunit interactions in multimeric proteins and in turn stabilizing protein oligomers [60-64]. Disruption of salt bridges thereby facilitates subunit movement [65] and thereby modulates stability of higher order oligomeric protein structures. Salt at high concentration is known to inhibit self assembly of individual subunits in protein oligomers [43, 66]. We therefore speculate salt mediated disruption of inter-subunit salt bridges in hRad52 structure can be the primary cause for the observed salt $(\mathrm{KCl})$ mediated monomerization of hRad52 aggregates. In this context, we looked for the presence of any salt bridges in hRad52 oligomer using the crystal structure data available for the homologous pairing domain of hRad52 undecamer, comprising of $\mathrm{N}$-terminal 212 amino acids as there is no crystal structure data available for full length hRad52 protein. While full length hRad52 protein is reported to form heptameric rings [23], hRad52 (N 1-212) truncated protein forms undecameric rings [24]. Modeling the heptameric fulllength hRad52 protein onto the crystal structure predicted for the undecameric truncated hRad52 (N1-212) protein, revealed presence of inter-subunit gaps wide enough to accommodate two Beta strands [24]. This implies that the inter-subunit electrostatic interactions and therefore, the overall structural topology predicted for the undecameric protein can also hold true for the heptameric full length protein. The comparable efficiencies of the truncated and full length hRad52 proteins in DNA-binding [13], self-assembly [67] and homologous pairing functions [18], can be explained by this possibility. The structural and functional homologies between these two proteins justify computing salt bridges using the crystal structure information for truncated hRad52 protein in order to explain salt-mediated monomerization of the full length hRad52 protein. Our computational analyses of hRad52 undecamer reveal an unusually high number of salt bridges between individual subunits. We further find uniqueness in the distribution of these salt bridges between subunits. Each subunit donates electrons ( $n$ ) to three other subunits, that are succeeding $(n+1)$, preceding $(n-1)$ and next to adjacent succeeding subunit (n+2) (Fig. 2 (i)). Conversely each subunit (n) in turn accepts electrons from three subunits that are not only its immediate neighbors $(n+1$ and $n-1)$ but also from (n-2) subunit (Fig. 2 (ii)). The overall inter-subunit connectivity can be described using the total number of electrostatic interactions (electron donation + electron acceptance) as depicted in (Fig. 2 (iii)). One can infer from this figure that hRad52 oligomeric structure is maintained to a large extent by electrostatic interactions $(\sim 40)$ between immediate subunits (example: A-B and K-A, (Fig. 2) (iii)), and to a lesser extent by electrostatic interactions $(\sim 7)$ between alternate subunits (example: A-C and J-A, (Fig. 2) (iii)). This second category of fewer electrostatic interactions is unidirectional, i.e. a subunit (n) always serves as a donor to the alternative subunit $(n+2)$. (For example, J with respect to A, (Fig. 2) (iii)). In light of existence of salt bridges between multiple subunits and their high numbers between specific subunits, we propose salt bridges as the stabilizing force between individual hRad52 subunits, in order to generate oligomeric hRad52 rings (Fig. 2 (iii)). Combining our observation of salt-mediated monomerization of hRad52 aggregates (Fig. 1(i) and (ii)), with the known solubilizing effect of salt on salt-bridges, we predict salt to solubilize the inter-subunit salt bridges, thereby bringing about monomerization of hRad52 aggregates (Fig. 4).

\section{Salt Aggregates hRad51 Molecules}

Addition of salt $(\mathrm{KCl})$ did not solubilize yet another oligomeric protein, hRad51 (Fig. 1(iii)), suggesting not all proteins are affected similarly by salt. Moreover, structural analysis of $P f \operatorname{Rad} 51$ revealed very few salt bridges between individual subunits (Fig. 3) in contrast to hRad52, reiterating the unique dense distribution of salt bridges to be a characteristic feature of higher order oligomeric proteins such as hRad52. We have employed the crystal structure data available for full length oligomeric Pyrococcus furiosus $\operatorname{Rad51}(\operatorname{Pf} \operatorname{Rad} 51)$ as the other existing crystal structure data available are those of Rad51 complexed with ADP/ ATP /ATP analogs/ ssDNA/ Potassium from Methanococcus voltae, Saccharomyces cerevisiae and humans [50-53, 35]. On the other hand the data used in our current study is that of full length polymeric Pf $\operatorname{Rad51}$ which reveals the atomic details crucial for quaternary assembly of RAD51 proteins into inactive heptameric rings and active DNA-bound filaments matching three-dimensional electron microscopy reconstructions [31]. Overall, the archaeal and human Rad51 proteins are close structural homologs that are distinct from RecA except for similarities within the conserved ATPase domain, implying that this domain forms the progenitor recombination unit, whereas the additional eukaryotic/ archaeal RAD51-N terminal domains and bacterial RecA-C terminal domain evidently evolved independently [31]. Sedimentation analyses for hRad51 while titrating with $\mathrm{KCl}$ at the same concentration range as for hRad52 revealed in contrast, increased aggregation of the protein upon addition of salt, as observed by increased recovery of protein in the pellet fractions (lanes p3-p6, Fig. 1(iii)). Salt mediated aggregation of proteins is known to be brought about by hydrophobic collapse where the hydrophobic residues at the interacting surfaces of monomers come together to result in larger structures [68-70]. This is most likely the case for hRad51, whose multimerization interface carries hydrophobic pockets [71], but not for hRad52 whose surface hydrophobicity is very low.

Further exploration of crystal structure data available for other oligomeric proteins such as 2-cys peroxiredoxin from human erythrocyrtes [PDB ID: 1QMV] which forms 
decameric ring structure, F-type $\mathrm{Na}^{+}$ATPase from Ilyobacter tartaricus [PDB ID: 1YCE] and Rabies viral protein [PDB ID: 2GTT] known to form undecameric ring organization, respectively, also revealed a dense unique network of salt bridges between individual subunits. However this property of uniqueness in terms of subunit connectivity varied from one protein to another. Exploring these differences is not within the scope of this study, but nevertheless, presence of high number of inter-subunit salt bridges appears to be a common structural feature of multimeric proteins, at least for ones forming decameric and undecameric oligomeric rings. This distinctive distribution of salt bridges between individual monomers could perhaps be the stabilizing factor in maintaining higher order structural organization in these proteins. Our observation bears physiological relevance, at least for hRad52, since the salt $(\mathrm{KCl})$ concentrations at which we observe these effects, match the intra cellular concentrations of $\mathrm{KCl}$.

\section{Functional Relevance of Aggregation Status of hRad51 and hRad52 Proteins}

At lower salt concentrations, hRad51 is predominantly in the monomerized form and hRad52 is predominantly, in a highly oligomerized form, and vice-versa at higher salt concentrations. This scenario where salt acts as a modulator of sedimentation status of these proteins makes perfect relevance in vivo, when we take into account the functional significance of their oligomerization status. It is well established that aggregated hRad52 can bind DNA ends efficiently than non-aggregated forms. Furthermore recent studies [22] have shown that Rad52 from S. cerevisiae contains a single functional NLS essential for its nuclear localization. The NLS in Rad52 is weak, as monomeric protein species that harbor this NLS are mainly located in the cytosol. In contrast, multimeric protein complexes wherein each subunit contains a single NLS sort efficiently to the nucleus. Based on the results it has been proposed that the additive effect of multiple NLS sequences in a Rad52 ringstructure ensure efficient nuclear localization of Rad52 [22]. It is thereby evident that conditions which favor hRad52 aggregation facilitate its efficient localization to the nucleus, better loading on to Double-strand Break (DSB) ends and thereby drive homologous recombination mediated DNA repair [18-20]. However in case of hRad51, the helical filamentous form is considered to be biologically inactive. The helical Rad51-nucleo protein filaments which result from polymerization of Rad51 monomers onto DNA substrate is considered as the active form [40-43, 45-49]. Previous studies have proposed that DNA mediated disassembly of hRad51 aggregates into monomers favor loading of hRad51 monomers on to DNA for hRad51-DNA nucleo-protein filament generation [72, 73]. Our study thus clarifies the role of $\mathrm{KCl}$ (salt) at respective concentration regimes in regulating the monomerization and oligomerization of hRad51 and hRad52 proteins, and thereby facilitating DNA repair.

\section{ABBREVIATIONS}

hRad52 = Human Rad52

$\mathrm{hRad} 51=$ Human $\operatorname{Rad} 51$
HR = Homologous Recombination

$\mathrm{KCl}=$ Potassium Chloride

NLS = Nuclear Localization Signal

ATP $=$ Adenosine Tri Phosphate

$\mathrm{ADP}=$ Adenosine Di Phosphate

$\mathrm{ATP} \gamma \mathrm{S}=$ Adenosine 5'-O-(3-thiotriphosphate)

AMP-PNP $=$ Adenosine 5'-[ $\beta, \gamma$-imido]triphosphate

\section{ACKNOWLEDGEMENTS}

We thank Swadhin Chandra Jana, TIFR, INDIA for his help in the structural aspects of this study.

\section{REFERENCES}

[1] Shen Z, Cloud KG, Chen DJ, Park MS. Specific interactions between the human RAD51 and RAD52 proteins. J Biol Chem 1996; 271: 148-52.

[2] Benson FE, Baumann P, West SC. Synergistic actions of Rad51 and Rad52 in recombination and DNA repair. Nature 1998; 391: 401-4.

[3] Milne GT, Weaver DT. Dominant negative alleles of RAD 52 reveal a DNA repair/recombination complex including Rad51 and Rad52. Genes Dev 1993; 7: 1755-65.

[4] Shinohara A, Ogawa T. Stimulation by Rad52 of yeast Rad51mediated recombination. Nature 1998; 391: 404-7.

[5] Sung P. Function of yeast Rad52 protein as a mediator between replication protein $\mathrm{A}$ and the Rad51 recombinase. J Biol Chem 1997; 272: 28194-7.

[6] New JH, Sugiyama T, Zaitseva E, Kowalczykowski SC. Rad52 protein stimulates DNA strand exchange by Rad51 and replication protein A. Nature 1998; 398: 407-10.

[7] Baumann P, West SC. Hetroduplex formation by human RAD51 protein: Effects of DNA end structure, hRPA and hRad52. J Mol Biol 1999; 291: 363-74.

[8] Park MS, Ludwig DL, Stigger E, Lee SH. Physical interaction between human RAD52 and RPA is required for homologous recombination in mammalian cells. J Biol Chem 1996; 271: 189969000.

[9] Jackson D, Dhar K, Wahl JK, Wold MS, Borgstahl GE. Analysis of the human replication protein A: Rad52 complex: evidence for crosstalk between RPA32, RPA70, Rad52 and DNA. J Mol Biol 2002; 321: 133-48.

[10] Rattray AJ, Symington LS. Multiple pathways for homologous recombination in Saccharomyces cerevisiae. Genetics 1995; 139: 45-6.

[11] Rattray AJ, Symington LS. Use of a chromosomal inverted repeat to demonstrate that the RAD51 and RAD52 genes of Saccharomyces cerevisiae have different roles in mitotic recombination. Genetics 1994; 138: 587-95.

[12] Mortensen UH, Bendixen C, Sunjevaric I, Rothstein R. DNA strand annealing is promoted by the yeast Rad52 protein. Proc Natl Acad Sci USA 1996; 93: 10729-34.

[13] Reddy G, Golub EI, Radding CM. Human Rad52 protein promotes single-strand DNA annealing followed by branch migration. Mutat Res 1997; 377: 53-9.

[14] Shinohara A, Shinohara M, Ohta T, Matsuda S, Ogawa T. Rad52 forms ring structures and co-operates with RPA in single-strand DNA annealing. Genes Cells 1998; 3: 145-6.

[15] Bi B, Rybalchenko N, Golub EI, Radding CM. Human and Yeast Rad52 proteins promote DNA strand exchange. Proc Natl Acad Sci USA 2004; 101: 9568-72.

[16] Kumar JK, Gupta RC. Strand exchange activity of human recombination protein Rad52. Proc Natl Acad Sci USA 2004; 101: 9562-7.

[17] Ranatunga W, Jackson D, Lloyd JA, Forget AL, Knight KL, Borgstahl GEO. Human RAD52 exhibits two modes of selfassociation. J Biol Chem 2001; 276: 15876-80. 
[18] Van Dyck E, Stasiak A, West SC. Binding of double-strand breaks in DNA by human Rad52 protein. Nature 1999; 398: 728-31.

[19] Van Dyck E, Hajibagheri NMA, Stasiak A, West SC. Visualization of human Rad52 protein and its complexes with hRad51 and DNA. J Mol Biol 1998; 284: 1027-38.

[20] Lloyd JA, Forget AL, Knight KL. Correlation of biochemical properties with the oligomeric state of Human Rad52 protein. J Biol Chem 2002; 277: 46172-8.

[21] Kagawa W, Kurumizaka H, Ikawa S, Yokoyama S, Shibata T. Homologous pairing promoted by the human Rad52 protein. J Biol Chem 2001; 276: 35201-8.

[22] Plate I, Albertsen L, Lisby M, et al. Rad52 multimerization is important for its nuclear localization in Saccharomyces cerevisiae. DNA repair 2008; 7: 57-66.

[23] Stasiak AZ, Larquet E, Stasiak A, et al. The human Rad52 protein exists as a heptameric ring. Curr Biol 2000; 10: 337-40.

[24] Kagawa W, Kurumizaka H, Ishitani R, et al. Crystal structure of the homologous pairing domain from the human $\operatorname{Rad} 52$ recombinase in the undecameric form. Mol Cell 2002; 10: 359-71.

[25] Sung, P. Catalysis of ATP-dependent homologous DNA pairing and strand exchange by yeast RAD51 protein. Science 1994; 265 : 1241-43.

[26] Shinohara A, Ogawa H, Ogawa T. Rad51 protein involved in repair and recombination in $S$. cerevisiae is a RecA-like protein. Cell 1992; 69: 457-470.

[27] Krejci L, Chen L, Van Komen S, Sung P, Tomkinson A. Mending the Break: Two DNA Double-Strand Break Repair Machines in Eukaryotes. Prog Nucleic Acid Res Mol Biol 2003; 74: 159-201.

[28] Bianco PR, Tracy RB, Kowalczykowski SC. DNA strand exchange proteins: a biochemical and physical comparison. Front Biosci1998; 3: 570-603.

[29] Story RM, Bishop DK, Kleckner N, Steitz TA. Structural relationship of bacterial RecA proteins to recombination proteins from bacteriophage T4 and yeast. Science 1993; 259: 1892-1896.

[30] Sandler SJ, Satin LH, Samra HS, Clark AJ. RecA-like genes from three archaean species with putative protein products similar to Rad51 and Dmc1 proteins of the yeast Saccharomyces cerevisiae. Nucleic Acids Res 1996; 24: 2125-32.

[31] Shin DS, Pellegrini L, Daniels DS, et al. Full-length archaeal Rad51 structure and mutants: mechanisms for RAD51 assembly and control by BRCA2. EMBO J 2003; 22: 4566-76.

[32] Baumann P, West SC. Role of the human RAD51 protein in homologous recombination and double-stranded-break repair. Trends Biol Sci 1998; 23: 247-251.

[33] Baumann P, Benson FE, West SC. Human Rad51 protein promotes ATP-dependent homologous pairing and strand transfer reactions in vitro. Cell 1996; 87: 757-766.

[34] Benson FE, Stasiak A, West SC. Purification and characterization of the human Rad51 protein, an analogue of E. coli RecA. EMBO J 1994; 23: 5764-71.

[35] Conway AB, Lynch TW, Zhang Y, et al. Crystal structure of a Rad51 filament. Nat Struct Mol Biol 2004; 11: 791-6.

[36] Yang S, Yu X, Seitz EM, Kowalczykowski SC, Egelman EH. Archaeal RadA protein binds DNA as both helical filaments and octameric rings. J Mol Biol 2001; 314: 1077-85.

[37] Granéli A, Yeykal CC, Robertson RB, Greene EC. Long-distance lateral diffusion of human Rad51 on double-stranded DNA. Proc Natl Acad Sci USA 2006; 103: 1221-6.

[38] Komori K, Miyata T, DiRuggiero J, et al. Both RadA and RadB are involved in homologous recombination in Pyrococcus furiosus. $\mathrm{J}$ Biol Chem 2000; 275: 33782-90.

[39] Passy SI, Yu X, Li Z, et al. Human Dmc1 protein binds DNA as an octameric ring. Proc Natl Acad Sci USA 1999; 96: 10684-8

[40] Masson JY, Davies AA, Hajibagheri N, et al. The meiosis-specific recombinase hDmc1 forms ring structures and interacts with hRad51. EMBO J 1999; 18: 6552-60.

[41] Sehorn MG, Sigurdsson S, Bussen W, Unger VM, Sung P. Human meiotic recombinase Dmc1 promotes ATP-dependent homologous DNA strand exchange. Nature 2004; 429: 433-7.

[42] Yu X, Jacobs SA, West SC, Ogawa T, Egelman EH. Domain structure and dynamics in the helical filaments formed by RecA and Rad51 on DNA. Proc Natl Acad Sci USA 2001; 98: 8419-24.

[43] Yoshioka K, Yumoto-Yoshioka Y, Fleury F, Takahashi M. pH and salt dependent self-assembly of Human Rad51 protein analyzed as fluorescence resonance energy transfer between labeled proteins. J Biochem 2003; 133: 593-7.

[44] Kinebuchi T, Kagawa W, Enomoto R, et al. Structural basis for octameric ring formation and DNA interaction of the human homologous- pairing protein Dmc1. Mol Cell 2004; 14: 363-74.

[45] Navadgi VM, Shukla A, Vempati RK, Rao BJ. DNA mediated disassembly of hRad51 and hRad52 proteins and recruitment of hRad51 to ssDNA by hRad52. FEBS J 2006; 273: 199-207.

[46] Ogawa T, Yu X, Shinohara A, Egelman EH. Similarity of the yeast RAD51 filament to the bacterial RecA filament. Science 1993; 259: 1896-99.

[47] Sung, P, Robberson, DL. DNA strand exchange mediated by a RAD51-ssDNA nucleoprotein filament with polarity opposite to that of RecA. Cell 1995; 82: 453-61.

[48] Baumann P, Benson FE, West SC. Human Rad51 protein promotes ATP-dependent homologous pairing and strand transfer reactions in vitro. Cell 1996; 87: 757-66.

[49] Ristic D, Modesti M, van der Heijden T, et al. Human Rad51 filaments on double- and single-stranded DNA: correlating regular and irregular forms with recombination function. Nucleic Acids Res 2005; 33: 3292-302.

[50] Pellegrini L, Yu DS, Lo T, et al. Insights into DNA recombination from the structure of a RAD51-BRCA2 complex. Nature 2002; 420: 287-293.

[51] Qian X, Wu Y, He Y, Luo Y. Crystal structure of Methanococcus voltae RadA in complex with ADP: hydrolysis-induced conformational change. Biochemistry 2005; 44: 13753-61.

[52] Wu Y, Qian X, He Y, Moya IA, Luo Y. Crystal Structure of an ATPase-active Form of Rad51 Homolog from Methanococcus voltae. J Biol Chem 2005; 280: 722-8.

[53] Wu Y, He Y, Moya IA, Qian X, Luo Y. Crystal structure of archaeal recombinase RAD A: a snapshot of it extended conformation. Mol Cell 2004; 15: 423-35

[54] Krejci L, Damborsky J, Thomsen B, Duno M, Bendixen C. Molecular dissection of interactions between Rad51 and members of the recombination-repair group. Mol Cell Biol 2001; 21: 966-76.

[55] Krejci L, Song B, Bussen W, Rothstein R, Mortensen UH, Sung P. Interaction with Rad51 is indispensable for recombination mediator function of Rad52. J Biol Chem 2002; 277: 40132- 41.

[56] Liu Y, Maizels N. Co-ordinated response of mammalian Rad51 and Rad52 to DNA damage. EMBO Rep 2000; 1: 85-90.

[57] Gasior SL, Olivares H, Ear U, Hari DM, Weichselbaum R, Bishop DK. Assembly of RecA-like recombinases: distinct roles for mediator proteins in mitosis and meiosis. Proc Natl Acad Sci USA 2001; 98: 8411-18.

[58] Kurumizaka H, Aihara H, Kagawa W, Shibata T, Yokoyama S. Human Rad51 amino acid residues required for Rad52 binding. J Mol Biol 1999; 291: 537-48.

[59] Navadgi VM, Dutta A, Rao BJ. Human Rad52 facilitates threestranded pairing that follows no strand exchange: A novel pairing function of the protein. Biochemistry 2003; 42: 15237-51.

[60] Rodgers KK, Sligar SG. Mapping electrostatic interactions in macromolecular associations. J Mol Biol 1991; 221: 1453-60.

[61] Ali MH, Imperiali B. Protein oligomerization: How and why. Bioorg Med Chem 2005; 13: 5013-20.

[62] Harrison OJ, Corps EM, Kilshaw PJ. Cadherin adhesion depends on a salt bridge at the N-terminus. J Cell Sci 2005; 118: 4123-70.

[63] Irimia A, Ebel C, Madern D, et al. The oligomeric states of Haloarcula marismortui Malate dehydrogenase are modulated by solvent components as shown by crystallographic and biochemical studies. J Mol Biol 2003; 326: 859-73.

[64] Radha Kishan KV, Newcomer ME, Rhodes TH, Guilliot SD. Effect of $\mathrm{pH}$ and salt bridges on structural assembly: Molecular structures of the monomer and intertwined dimer of the Eps8 SH3 domain. Protein Sci 2001; 10: 1046-55.

[65] Yu B, Paroutis P, Davidson AR, Howell PL. Disruption of a salt bridge dramatically accelerates subunit exchange in duck delta2 crystallin. J Biol Chem 2004; 279: 40972-9.

[66] Chambers JW, Kearns MT, Morris MT, Morris JC. Assembly of heterohexameric Trypanosome hexokinases reveals that hexokinase 2 is a regulable enzyme. J Biol Chem 2008; 283: 14963-70.

[67] Shen Z, Peterson SR, Comeaux JC, et al. Self-association of human RAD52 protein. Mutat Res 1996; 364: 81-9. 
[68] Blokzijl W, Engberts, JBFN. Hydrophobic effects. Opinions and Facts. Angew Chem Int Ed Engl 2003; 32: 1545-79.

[69] Curtis RA, Steinbrecher C, Heinemann M, Blanch HW, Prausnitz JM. Hydrophobic forces between protein molecules in aqueous solutions of concentrated electrolyte. Biophys Chem 2002; 98: 24965.

[70] Samontha A, Nipattamanon C, Shiowatana J, Siripinyanond A. toward better understanding of salt-induced hen egg white protein aggregation using field-flow fractionation. J Agric Food Chem 2008; 56: 8809-14.
[71] Lo T, Pellegrini L, Venkitaraman AR, Blundell TL. Sequence fingerprints in BRCA2 and RAD51: implications for DNA repair and cancer. DNA Repair 2003; 2: 1015-28.

[72] West SC. Molecular views of recombination proteins and their control. Nat Rev Mol Cell Biol 2003; 4: 435-45.

[73] Sauvageau S, Stasiak AZ, Banville I, Ploquin M, Stasiak A, Masson JY. Fission yeast Rad51 and Dmc1, two efficient DNA recombinases forming helical nucleoprotein filaments. Mol Cell Biol 2005; 25: 4377-87.

(C) Balakrishnan et al.; Licensee Bentham Open.

This is an open access article licensed under the terms of the Creative Commons Attribution Non-Commercial License (http: //creativecommons.org/licenses/by$\mathrm{nc} / 3.0 /$ ), which permits unrestricted, non-commercial use, distribution and reproduction in any medium, provided the work is properly cited. 